\title{
An analysis of perceived usefulness of Google Scholar by the postgraduate students of the University of Ilorin, Nigeria
}

\begin{tabular}{|c|c|}
\hline \multicolumn{2}{|c|}{$\begin{array}{l}\text { Authors: } \\
\text { Adeyinka Tella }{ }^{1,2} \\
\text { Michael Oyewole } \\
\text { Adedeji Tella }^{3}\end{array}$} \\
\hline \multicolumn{2}{|c|}{$\begin{array}{l}\text { Affiliations: } \\
{ }^{1} \text { Department of Library and } \\
\text { Information Science, } \\
\text { University of Ilorin, Nigeria }\end{array}$} \\
\hline \multicolumn{2}{|c|}{$\begin{array}{l}{ }^{2} \text { Department of Information } \\
\text { Science, University of South } \\
\text { Africa, South Africa }\end{array}$} \\
\hline \multicolumn{2}{|c|}{$\begin{array}{l}{ }^{3} \text { Department of Teacher } \\
\text { Education, University of } \\
\text { Ibadan, Nigeria }\end{array}$} \\
\hline \multicolumn{2}{|c|}{$\begin{array}{l}\text { Corresponding author: } \\
\text { Adeyinka Tella, } \\
\text { tellayinkaedu@yahoo.com }\end{array}$} \\
\hline \multicolumn{2}{|c|}{$\begin{array}{l}\text { Dates: } \\
\text { Received: } 19 \text { Sept. } 2016 \\
\text { Accepted: } 12 \text { Feb. } 2017 \\
\text { Published: } 23 \text { May } 2017\end{array}$} \\
\hline \multicolumn{2}{|c|}{$\begin{array}{l}\text { How to cite this article: } \\
\text { Tella, A., Oyewole, M. \& Tella, } \\
\text { A., 2017, 'An analysis of } \\
\text { perceived usefulness of } \\
\text { Google Scholar by the } \\
\text { postgraduate students of the } \\
\text { University of Ilorin, Nigeria', } \\
\text { South African Journal of } \\
\text { Information Management } \\
\text { 19(1), a793. https://doi. } \\
\text { org/10.4102/sajim.v19i1.793 }\end{array}$} \\
\hline \multicolumn{2}{|c|}{$\begin{array}{l}\text { Copyright: } \\
\text { (c) 2017. The Authors. } \\
\text { Licensee: AOSIS. This work } \\
\text { is licensed under the } \\
\text { Creative Commons } \\
\text { Attribution License. }\end{array}$} \\
\hline \multicolumn{2}{|l|}{ Read online: } \\
\hline 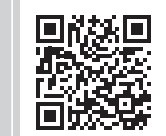 & $\begin{array}{l}\text { Scan this QR } \\
\text { code with your } \\
\text { smart phone or } \\
\text { mobile device } \\
\text { to read online. }\end{array}$ \\
\hline
\end{tabular}

Background: Google Scholar provides user-friendly information resources and is very effective in finding information to satisfy various information needs, especially by the research students. However, despite its usefulness in satisfying information and research needs of the research students and researchers as a whole, the lingering effect associated with it is drawing students away from libraries. Similarly, there have been limited studies conducted to examine the level of perceived usefulness of Google Scholar by the graduate students, particularly in the context of the University of Ilorin, Nigeria.

Objectives: The study examined the perceived usefulness of Google Scholar by the postgraduate students of the University of Ilorin, Nigeria.

Methods: A total of 223 postgraduate students selected through simple random sampling from 15 faculties that made up the University formed the sample for the study. A survey approach was adopted using a questionnaire to gather data from respondents. Six research questions were developed to guide the study.

Results: The results demonstrate that the majority of respondents strongly agreed and agreed that they were aware of Google Scholar and usually used it. However, the respondents were not satisfied with Google Scholar as it does not either speed up their research or make their research easier. Google Scholar was considered useful because it covers broad topics in the area of interest and usually provides relevant articles related to the respondents' search done through this platform.

Conclusion: The study concluded by recommending, among others, that there is a need for orientation programmes to enhance the use of Google Scholar via the university library.

\section{Introduction}

Information is very essential in our day-to-day activities. It is needed for a variety of reasons which mostly involves solving problems. When faced with a problem, we search for information that will help us proffer solutions to remediate the problems. As Belkin and Croft (1992) pointed out, a search usually commences with a problem and the need to solve it. They explained that the gap between the two is the need for information, which now leads to information seeking.

The need for such information makes us use the library and other information repositories to find solutions to problems. Such problems may be academic, social, economic, health or financial. The need to use information in analysing, correcting, informing and solving problems is almost an unquenchable thirst as people demand information on a very large scale. In different walks of life, information is needed to get ahead of competition, market resources and to make informed decisions.

With the invention of technological devices such as cell phones, computers and others, information has become mobile in terms of accessibility. The Internet, for instance, is a huge repository of information that one can access anytime and anywhere for information. Internet is the interconnection of different networks and servers to form a giant network. The Internet has been defined as a network of networks. As the network includes different devices, it is easy to access vast amounts of information. There are thousands of websites which vary with the information they provide. While some provide academic information, others provide economic information, social information and so on. 
Before the advent of the Internet, the library has been in existence as a veritable source for information. However, not everybody is capable of using the library and the materials in it. This has resulted in complications in the area of information retrieval, as users may either be far away from where a library is or been unable to access appropriate materials needed because of limitations in the searching strategy.

The revolution of the Internet has resulted in the availability of so many sources to access information. For instance, Google Scholar provides a new technique of locating a relevant article on a particular subject. This is done by identifying a subsequent publication that cites or references a previously published work. Features of Google Scholar identified by Noruzi (2005) include opportunity for researchers to trace interconnections among authors, citing articles on a relevant topic and determine the extent to which others cite a specific article, as it has a 'cited by' feature.

Information is essential to society. With the ever increasing need for information, especially in the academic world, there is a need for students to search for information to solve problems. The issue of information needs to be addressed as information is the bedrock for the survival and sustenance of the society. Lack of information in a society can lead to faulty decisions and decline in economic growth and academics. Once students are given tasks to perform, they need information to perform such tasks. This need will motivate research which will in turn produce a result which may be positive or negative depending on the information obtained. The need for information is a global issue as every society depends on it. The academic world hangs on information as it is used to carry out analysis and study data so as to make informed choices. The medium of accessing information is as important as information itself. When there is a need for information, the information seeker has to use a medium of information retrieval. This medium can be a library or other online applications such as Google Scholar.

Various studies by librarians and academics have demonstrated that Google Scholar provides friendly information resources to users; however, the lingering effect associated with this is drawing students away from libraries. There is no doubt that Google Scholar is very effective in finding information to satisfy various information needs, especially for research students. However, despite the usefulness of Google Scholar in satisfying information and research needs of research students and researchers as a whole, there have been limited studies conducted to examine the level of perceived usefulness of Google Scholar by the research students (postgraduate students), particularly in the context of the University of Ilorin, Nigeria.

The result of this research is significant in several ways. It will help in understanding the perspective of the postgraduate students of the University of Ilorin on the usefulness of the Google Scholar platform. The research will also create awareness on the part of other institutions' postgraduate students on how to effectively use Google Scholar for research. The outcomes from the research are expected to lead to a change of attitudes towards information searching as users will not be constrained to the use of a library. Also, it will help understand the advantages and reasons for using Google Scholar by postgraduate students.

\section{Objective of the study}

The broad objective of this study is to examine the perceived usefulness of Google Scholar by the postgraduate students of the University of Ilorin, Nigeria. The specific objectives of the study are to:

- identify the level of awareness of postgraduate students of the University of Ilorin towards the use of Google Scholar;

- examine the perception of postgraduate students of the University of Ilorin on the ease of use of Google Scholar;

- determine the perceived usefulness of Google Scholar to postgraduate students of the University of Ilorin;

- find out the degree of comprehensiveness and trust on information found on Google Scholar by the postgraduate students of the University of Ilorin;

- ascertain the satisfaction level of postgraduate students of the University of Ilorin with Google Scholar platform;

- identify the continued intention of postgraduate students of the University of Ilorin towards the use of Google Scholar platform.

\section{Research questions}

The following questions guided this study:

- What is the level of awareness of postgraduate students of the University of Ilorin towards the use of Google Scholar?

- What is the perception of postgraduate students of the University of Ilorin towards the ease of use of Google Scholar?

- What is the perceived usefulness of Google Scholar to postgraduate students of the University of Ilorin?

- What is the degree of comprehensiveness and trust on information found on Google Scholar by the postgraduate students of the University of Ilorin?

- What is the satisfaction level of postgraduate students of the University of Ilorin with Google Scholar platform?

- What is the intention of postgraduate students of the University of Ilorin towards the use of Google Scholar?

\section{Literature review}

Google Scholar describes a freely accessible search engine which enables users to search for print and electronic copies of published articles. The search engine searches various sources such as academic publishers, universities and preprint depositories by looking for articles, thesis and dissertations, citations and journals (Mikki 2009). It is a database that contains different databases majorly used in searching for text and scholarly articles that can either be digital or printed copies. According to Mikki, Google Scholar does this 
searching by linking with databases of some other academic institutions which it partners with. The coverage of Google Scholar is interdisciplinary because it covers almost all subjects in all disciplines. This allows it to generate a broad result when queries are entered into it. By linking with other databases, Google Scholar accesses their scholarly articles and publications, indexes and cites them on its own platform (Jasco 2005).

Since Google Scholar is a freely accessible search engine, it is being patronised by information users and libraries who cannot afford to increase their budget for information search facilities. Considering this factor, a lot of reviews have been done by librarians to check the credibility and reliability of the information obtained through Google Scholar (Adlington \& Benda 2006; Callicott \& Vaughn 2005). This also leads to questions regarding the efficiency and experience of users accessing Google Scholar, to understand their reactions to the search engine in assisting their research work.

\section{Empirical framework}

Shen (2012) conducted a study on the frequency of graduate students' usage of Google Scholar and the contributing factors to its adoption. The findings demonstrated that $45 \%$ of those who had used Google Scholar indicated its linkage to full text articles via the customised library link. On average, respondents found Google Scholar easy to use ( $M=4.09$ out of 5) and access $(M=3.86)$. Respondents also perceived Google Scholar as a useful resource for research because it enhanced their search effectiveness. On the other hand, respondents were not emphatic about whether or not they always found what they are searching for using Google Scholar or whether or not enough resources are available on it for their research. Nonetheless, most of the respondents were still convinced they made the right choice to use Google Scholar $(M=3.94)$. The results further revealed several factors that strongly influence graduate students' intention to use Google Scholar. These are perceived usefulness of Google Scholar, sense of loyalty, and perceived ease of use. The findings provide useful insights for librarians seeking to understand graduate students' perception of Google Scholar and practical implications on how best Google Scholar can be promoted to graduate students.

Cothran (2011) examined a quantitative analysis of Google Scholar acceptance and use among graduate students. By applying a web-based survey questionnaire, the results revealed that $73 \%$ of the respondents reported having used Google Scholar at least once before. However, $45 \%$ of those who had used Google Scholar reported its linkage to full text articles. The results revealed further that on average, respondents perceived Google Scholar as easy to use and easy to access. Hamid and Asadi (2010) investigated the role of Google Scholar in the information seeking behaviour of scientists including physicists and astronomers from the Department of Physics and Astronomy at the University College, London. A mixed method approach was adopted using semi-structured interview items, a questionnaire and information-event cards as instruments for the collection of data from 114 respondents. The results demonstrated that there is increasing reliance of scientists on general search engines, especially Google, for locating scholarly articles. Based on the findings, the study concluded that the increasing awareness of the large quantity of scholarly articles available and searchable via Google by the scientists make them rely on it for finding scholarly publications.

Neuhaus, Neuhaus and Asher (2008) conducted a study to determine the degree of Google Scholar adoption by academics. The authors analysed the frequency of Google Scholar appearances on 948 campus and library websites and established the link resolution between Google Scholar and library resources. The results showed that a positive correlation exists between the implementation of Google Scholar link resolution and the degree of Google Scholar adoption.

In a comparative analysis of Google Scholar interface and search engine Scirus conducted by Felter (2005), it was reported that although Scirus has a sophisticated interface, most researchers preferred the simplicity of Google and would more likely opt for it than other more capable and complicated databases. Henderson (2005) assessed the search capabilities of Google Scholar and reported a ranking bias towards older articles that has the passage of time been cited over a number times. The results also showed that Google Scholar lacked the standard search features such as 'similar pages' and 'did you mean' features for alternative spellings. Golderman and Connolly (2007) identified the compatibility of Google Scholar with bibliographic software such as Endnote and Reference works. However, they faulted Google Scholar for failing to include search histories, alert services, and utilities for sorting, marking and saving results.

Kousha and Thelwall (2006) in a study on Google Scholar citations indicated that there is a strong relationship between Google Scholar and ISI Web of Science for biology, computer science and physics journals. They also found moderate but statistically significant correlations between citations from Google Scholar and ISI for journals in sociology and psychology, education, chemistry and economics. The results showed further significant correlations between Google Scholar citation counts and ISI Journal Impact Factors. Noruzi (2006) in a related study tested the citation counts for 36 frequently cited papers in webometrics of both Google Scholar and Web of Science. The findings indicated that Google Scholar identified more citations than Web of Science for all but three of the articles. In another similar study, Bakkalbasi and Bauer (2005) compared citation counts in Google Scholar, Web of Science and Scopus for 1985 and 2000 using articles from the Journal of the American Society of Information Science and Technology (JASIST). The finding revealed that while Web of Science returned the most citation counts for 1985, Google Scholar tallied with the highest citation counts for all JASIST articles published in 2000. 
Jasco (2005) in another study that compares Google Scholar, Scopus and Web of Science examined the relative coverage of the most cited papers from the journal Current Science. The report demonstrated that in a total citation count of 30 articles, both Web of Science and Scopus outperformed Google Scholar by a three to one margin.

Furthermore, Bakkalbasi et al. (2006) assessed the number of citing references on articles in the fields of oncology and condensed matter physics generated by Web of Science, Scopus and Google Scholar. They found that Web of Science and Scopus returned more citing references than Google Scholar, but Google Scholar returned the highest number of unique references. The study concluded that none of the three resources clearly outperformed the others and that a researcher relying on just one or even two of the resources might fail to find all references. In their comparative bibliometric study of Web of Science, Scopus, and Google Scholar, Meho and Yang (2007) reported that in the field of Library and Information Science, Google Scholar provides citations from a broader array of sources than either Scopus or Web of Science. However, many of the additional sources were from low-impact journals and conference proceedings.

Another comparative analysis of contents from 47 different databases with Google Scholar was conducted by Neuhaus et al. (2006). The results revealed that database contents inclusion in Google Scholar varies considerably from one database to another database and from one discipline to another discipline. A great variation was discovered between Google Scholar's coverage of freely accessible databases and restricted access databases. Based on the finding, the study concluded that Google Scholar coverage score was greater for databases within science, medicine and social science discipline categories. The drawbacks of Google Scholar identified included the lack of coverage of social science and humanities databases and a bias towards English language.

From the extant literature, it is evident that the use of search engines, particularly Google Scholar, is now common among undergraduate and graduate students. However, most of the available studies on the subject were conducted in the developed countries where there is a stable network and Internet connectivity $(24 \times 7)$. Graduates, undergraduates, scholars and researchers in developing countries also rely on Google Scholar in the conduct of their research; unfortunately, studies on the usefulness of this tool by these sets of users in developing countrie, particularly among the postgraduate students in Nigeria, are limited.

\section{Methodology \\ Research design}

A survey approach was adopted in the conduct of this research. This was to be able to reach out to a sizeable number of respondents. Similarly, survey has been the dominant approach used in previous related studies (e.g. Jasco 2005; Neuhaus et al. 2006).

\section{Sample and sampling technique}

The target population used in the study is the postgraduate students of the University of Ilorin, Nigeria. As at the 2015-2016 session, this population spread across 15 faculties that made up the postgraduate scheme of the university. The faculties are Faculty of Agriculture, Faculty of Arts, Faculty of Basic Medical Sciences, Faculty of Social Sciences, Faculty of Management Sciences, Faculty of Clinical Sciences, Faculty of Communication and Information Sciences, Faculty of Education, Faculty of Engineering and Technology, Faculty of Law, Faculty of Pharmaceutical Sciences, Faculty of Life Science, Faculty of Veterinary, Faculty of Physical Sciences and Faculty of Environment.

This study adopted the stratified sampling technique because the population has already been divided into natural strata (faculties). The population has homogenous groups known as strata. Each stratum is a representation of a faculty (postgraduate) in the university, bringing the total to 15 strata. Using simple random sampling method, the sample was drawn from the 15 strata. Fifteen respondents (postgraduate students) were selected from each of the faculties of the university, making a total of 225 postgraduate students who represent the sample for the study.

\section{Data collection instrument}

The main instrument used was a researcher-designed questionnaire tagged 'Perceived Usefulness of Google Scholar Questionnaire'. The questionnaire consists of a list of items relating to the research questions mentioned and the objectives. The questionnaire was divided into three sections. Section A requested the respondents' demography which includes age, gender and faculty. Section B contained the items that focus on each of the variables in the study and was further divided into parts. Each part focused on a variable in the objectives. For instance, Part 1 featured items on level of awareness of postgraduate students of the University of Ilorin towards the use of Google Scholar. Part B featured items on the perception of postgraduate students on the ease of use of Google Scholar. Part C featured the perceived usefulness of Google Scholar by postgraduate students. Part $\mathrm{D}$ featured items on the degree of trust in information found on Google Scholar by the postgraduate students. Part E featured items on the satisfaction level of the postgraduate students with Google Scholar platform, while Part F featured items on the intention of the postgraduate students towards the use of the Google Scholar platform. A Likert-type scale format with Strongly Agreed (SA), Agreed (A), Disagreed (D) and Strongly Disagreed (SD) was adopted for all items in Parts A-F of the instrument.

\section{Validity of the instrument}

In order to ensure the content and construct validity, the instrument was given to two specialists to scrutinise and check for appropriateness of language to examine its suitability for data collection before administering the instruments to the respondents. The suggestions made 
by the experts led to modifications of some items in the instrument before it was finally administered.

\section{Reliability of the instrument}

To study the reliability of the instrument, a split-half reliability method was adopted. This method involves administering the instrument to a set of 20 respondents outside the envisaged population. The responses collected were divided into two equal halves, while the data collected were subjected to Pearson's product moment correlation. The correlation coefficient returned an $r$ of 0.88 . This indicated that the instrument was highly reliable for data collection on the study.

\section{Data collection procedures}

The questionnaire on the perceived usefulness of Google Scholar by postgraduate students of the University of Ilorin was administered by the researcher to the respondents. The questionnaire was administered during the rainy semester of the 2015-2016 academic session. This is because that is the time when they could be easily reached. With the assistance of colleagues in different faculties of the university, the questionnaires administration exercise was an itch-free one and consequently resulted in no attrition rate. Fifteen copies of questionnaires were administered to respondents in each faculty, making a total of 225 copies. Out of the 225 , a total of 223 copies of the questionnaire were returned properly filled and were thus good for data analysis given, representing a $99.1 \%$ return rate.

\section{Methods of data analysis}

Data analysis was carried out with the use of appropriate statistical methods. The researcher collated data by collecting responses on Sections A, B, C, D, E and F. The analysis was conducted using item by item analysis; the data collected were analysed with the use of simple percentage and frequency count. The data collected were coded using SPSS Version 21.0.

\section{Data presentation and analysis and results}

Table 1 shows that out of the 223 respondents, 142 (63.7\%) were males while $81(36.3 \%)$ were females. With this, it is clear that the population of male respondents is larger than the female population. On the age distribution of the respondents, the table also indicates that 60 (26.9\%) respondents fall within the ages of 18-23 years; this is followed by the respondents within the ages of 24-29 years with $122(54.7 \%)$. Next to it is the respondents who were between the ages of 30 years and above with 41 (18.4\%). With the results presented, it is clear that students within the age range of 30 years and above represent the majority of the respondents. The results also revealed the statistics of the faculties in relation to the respondents. The lowest number of respondents was from the faculty of Basic Medical Sciences with 13 (5.8\%), whereas the other 14 faculties had $15(6.7 \%)$ respondents each.

\section{Research Question 1}

What is the level of awareness of postgraduate students of the University of Ilorin towards the use of Google Scholar?

Table 2 reveals that out of 223 (100\%) students sampled, 21 (9.4\%) respondents strongly agree to have awareness, 102 (45.7\%) respondents agree to being aware, 71 (31.8\%) disagree to being aware, while 29 (13.1\%) strongly disagree to being aware. This shows that the majority of respondents are aware of Google Scholar. Of all the respondents, only 34 (15.3\%) strongly agree that they can use Google Scholar. A total of 98

\begin{tabular}{lcc} 
TABLE 1: Demography of the respondents. & & \\
\hline Demographics & Frequency & $\%$ \\
\hline Gender & & \\
Male & 142 & 63.7 \\
Female & 81 & 36.3 \\
Total & 223 & 100 \\
Age & & \\
18-23 & 60 & 26.9 \\
24-29 & 122 & 54.7 \\
30 above & 41 & 18.4 \\
Total & 223 & 100 \\
Faculties & & \\
Agricultural science & 15 & 6.7 \\
Arts & 15 & 6.7 \\
Basic medical science & 13 & 5.8 \\
Clinical sciences & 15 & 6.7 \\
Communication and information sciences & 15 & 6.7 \\
Education & 15 & 6.7 \\
Engineering and technology & 15 & 6.7 \\
Environment & 15 & 6.7 \\
Law & 15 & 6.7 \\
Life sciences & 15 & 6.7 \\
Management sciences & 15 & 6.7 \\
Pharmaceutical sciences & 15 & 6.7 \\
Physical sciences & 6.7 \\
Social sciences & 6.7 \\
Veterinary & 15 & 6.7 \\
\hline Total & 15 & \\
\hline & & \\
\hline
\end{tabular}

TABLE 2: The level of awareness of Google Scholar.

\begin{tabular}{|c|c|c|c|c|c|c|c|c|}
\hline \multirow[t]{2}{*}{ Awareness of Google Scholar } & \multicolumn{2}{|c|}{ SA } & \multicolumn{2}{|c|}{ A } & \multicolumn{2}{|c|}{ D } & \multicolumn{2}{|c|}{ SD } \\
\hline & $n$ & $\%$ & $n$ & $\%$ & $n$ & $\%$ & $n$ & $\%$ \\
\hline 1. I am aware of Google Scholar & 21 & 9.4 & 102 & 45.7 & 71 & 31.8 & 29 & 13.1 \\
\hline 2. I can make use of Google Scholar & 34 & 15.3 & 98 & 43.9 & 84 & 37.7 & 7 & 3.1 \\
\hline 3. I make use of Google Scholar via a library & 1 & 0.4 & 6 & 2.7 & 195 & 87.5 & 21 & 9.4 \\
\hline 4. I access Google Scholar via other medium & 7 & 3.1 & 193 & 86.6 & 23 & 10.3 & - & 0 \\
\hline 5. I use Google Scholar for research purposes & 43 & 19.3 & 106 & 47.5 & 67 & 30.1 & 7 & 3.1 \\
\hline
\end{tabular}

SA, strongly agree; A, agreed; D, disagreed; SD, strongly disagreed. 
(43.9\%) respondents agree to making use of Google Scholar, $84(37.7 \%)$ respondents disagree to having made use of Google Scholar, while 7 (3.1\%) strongly disagree to making use of Google Scholar. This shows that the majority of respondents strongly agree and agree with the fact that they can make use of Google Scholar.

As regards accessing Google Scholar via the library, one (0.4\%) of the respondents attests that they strongly agree they often use Google Scholar through the library, while six (2.7\%) of the respondents agree they often use Google Scholar via the library. However, 195 (87.5\%) respondents disagree to have used Google Scholar via the library, while 21 (9.4\%) strongly disagree. With this, it shows that respondents rarely use Google Scholar via the library. Furthermore, the results show the ratio of respondents that make use of Google Scholar via other mediums. A total of 7 (3.1\%) respondents strongly agree using Google Scholar via other mediums, and 193 (86.6\%) respondents agree to using Google Scholar via other mediums. From this, it is clear that the percentage of respondents using Google Scholar via other means trumps those using it via the library. With this result, it is clearly shown that the majority of respondents use Google Scholar via other mediums.

Of the $223(100 \%)$ respondents, $43(19.3 \%)$ strongly agree to using Google Scholar for research purposes, while 106 (47.5\%) respondents agree to making use of Google Scholar for research purposes. However, 67 (30.1\%) respondents disagree to using Google Scholar for research purposes, while 7 (3.1\%) respondents strongly disagree. This shows that the majority of respondents use Google Scholar for research purposes.

\section{Research Question 2}

What is the perception of postgraduate students of the University of Ilorin towards the ease of use of the Google Scholar?

Table 3 shows the perceived ease of use of Google Scholar among the postgraduate students of the University of Ilorin. A total of $22(9.9 \%)$ respondents strongly agree to finding Google Scholar easy to use; 121 (54.2\%) respondents also agree to finding Google Scholar easy to use. However, 71 $(31.8 \%)$ respondents disagree that Google Scholar is easy to use, while $9(4.1 \%)$ strongly disagreed. With these results, it is clear that the majority of respondents agree that Google Scholar is easy to use. With regards to the mental effort required in interacting with Google Scholar, 37 (16.6\%) respondents strongly agree that interacting with Google Scholar does not require a lot of mental effort, while 122
$(54.7 \%)$ respondents agree with the statement. However, 56 (25.1\%) respondents disagree with the statement that Google Scholar does not require a lot of mental effort in its interaction, while $8(3.6 \%)$ strongly disagree. This shows that the majority of respondents agree that interacting with Google Scholar does not require a lot of mental effort.

Table 3 also confirms that $17(7.6 \%)$ respondents strongly agree to finding it easy getting what they want on Google Scholar, while 114 (51.1\%) agree to finding it easy getting what they want on Google Scholar. However, 81 (36.4\%) respondents disagree to finding it easy to get what they want on Google Scholar, while 11 (4.9\%) respondents strongly disagree. With this, it shows that the majority of respondents find it easy to get what they want on Google Scholar.

However, 46 (20.6\%) respondents strongly agree to finding it easy to understand the terms used in Google Scholar; $86(38.6 \%)$ respondents also agree with this statement. The remainder of the respondents, however, decided otherwise, with 79 (35.4\%) of them disagreeing and 12 (5.4\%) strongly disagreeing to finding it easy to understand the terms used in Google Scholar. This shows that the number of respondents who find it easy to understand the terms used in Google Scholar is slightly more than those who do not.

A total of $27(12.1 \%)$ respondents strongly agree to finding it easy to learn to use Google Scholar and 103 (46.2\%) also agree to finding it easy to learn to use Google Scholar. However, 77 (34.5\%) of the remaining respondents disagree to finding it easy to learn to use Google Scholar, while 16 $(7.2 \%)$ strongly disagree with the statement. This shows that the majority of respondents find it easy to learn to use Google Scholar.

\section{Research Question 3}

What is the perceived usefulness of Google Scholar to the postgraduate students of the University of Ilorin?

The results in Table 4 reveal that of the $223(100 \%)$ respondents, only $36(16.1 \%)$ strongly agree that using Google Scholar enables them to accomplish their tasks more quickly and $74(33.2 \%)$ agree to the same statement. However, $92(41.3 \%)$ respondents disagree and $21(9.4 \%)$ strongly disagree. This shows that a slight majority of the respondents agree that Google Scholar enables them to accomplish their tasks faster. In terms of research, 29 (13.1\%) respondents

TABLE 3: Perception of postgraduate students on the ease of use of Google Scholar.

\begin{tabular}{|c|c|c|c|c|c|c|c|c|}
\hline \multirow[t]{2}{*}{ Perceived ease of use } & \multicolumn{2}{|c|}{ SA } & \multicolumn{2}{|c|}{ A } & \multicolumn{2}{|c|}{ D } & \multicolumn{2}{|c|}{ SD } \\
\hline & $n$ & $\%$ & $n$ & $\%$ & $n$ & $\%$ & $n$ & $\%$ \\
\hline 1. I find Google Scholar is easy to use & 22 & 9.9 & 121 & 54.2 & 71 & 31.8 & 9 & 4.1 \\
\hline 2. Interacting with Google Scholar does not require a lot of mental effort & 37 & 16.6 & 122 & 54.7 & 56 & 25.1 & 8 & 3.6 \\
\hline 3. I find it easy to get Google Scholar to do what I want it to do & 17 & 7.6 & 114 & 51.1 & 81 & 36.4 & 11 & 4.9 \\
\hline 4. I find it easy to understand the terms used throughout Google Scholar & 46 & 20.6 & 86 & 38.6 & 79 & 35.4 & 12 & 5.4 \\
\hline 5. Learning to use Google Scholar is easy for me & 27 & 12.1 & 103 & 46.2 & 77 & 34.5 & 16 & 7.2 \\
\hline
\end{tabular}

SA, strongly agree; A, agreed; D, disagreed; SD, strongly disagreed. 
strongly agree to Google Scholar making it easier for them to do their research. A total of 52 (23.3\%) respondents agree that Google Scholar makes their research work easier, but $93(41.7 \%)$ respondents disagree and $49(21.9 \%)$ of them strongly disagree. This statistics shows that the majority of respondents disagree that Google Scholar makes their research easier.

The results also confirm that of the $223(100 \%)$ respondents, $25(11.2 \%)$ strongly agree and $98(43.9 \%)$ agree that using Google Scholar enhances their searching effectiveness. However, $84(37.7 \%)$ respondents disagree and 16 (7.2\%) strongly disagree. The numbers of respondents who agree that using Google Scholar enhances their searching effectiveness are almost the same as those who do not. However, those who agree are slightly more than those who do not. For any research to be useful, there is a need for finding relevant articles to assist the research. The table shows that $31(13.9 \%)$ respondents strongly agree to finding many relevant articles with one search on Google Scholar. A total of $93(41.7 \%)$ respondents also agree with this statement, but $77(34.5 \%)$ disagree and $22(9.9 \%)$ strongly disagree to finding many relevant articles on Google Scholar with one search. This statistic shows that a slight majority of the respondents agree to find many relevant articles on Google Scholar with one search. The results also show that 31 (13.9\%) respondents strongly agree and $96(43.1 \%)$ respondents agree that the resources found in Google Scholar relate well to their research. However, 54 (24.2\%) and 52 (23.3\%) respondents disagree and strongly disagree, respectively, that the resources found in Google Scholar relate well with their research. With this, it shows that a slight majority of the respondents agree that resources found in Google Scholar relate well with their research.

\section{Research Question 4}

What is the degree of comprehensiveness given to information found on Google Scholar by the postgraduate students of the University of Ilorin?
Table 5 shows how comprehensive Google Scholar is among the postgraduate students of the University of Ilorin. The table shows that $26(11.6 \%)$ respondents strongly agree to easily understanding the resources found on Google Scholar while 103 (46.2\%) respondents agree with the same statement. However, 78 (34.9) respondents disagree while 16 (7.2\%) strongly disagree to understanding the resources found on Google Scholar. With this, it is obvious that majority of the respondents find it easy to understand resources on Google Scholar. The table also shows that $106(47.5 \%)$ respondents strongly agree and $28(12.6 \%)$ of them agree that Google Scholar has enough resources for their study. Of the respondents, $74(33.2 \%)$ and $15(6.7 \%)$ however disagree and strongly disagree, respectively, with the statement that Google Scholar has enough resources for their study. This shows that the majority of respondents agree that Google Scholar has enough resources for their study. In terms of the coverage of a wide range of topics in particular area of interest, $45(20.2 \%)$ respondents strongly agree, 91 (40.8\%) agree, $74(33.2 \%)$ disagree and $13(5.8 \%)$ strongly disagree that Google Scholar covers a wide range of topics in their particular area of interest; with this, it is clear that the majority of respondents agree that Google Scholar covers a wide range of topics in their particular area of interest. Also, 16 (7.2\%) of the respondents strongly agree to often find exactly what they search for on Google Scholar. With 34 (15.2\%) agreeing to this statement also, the remainder however do not share the same view. Of the respondents, 126 (56.5\%) disagree and $47(21.1 \%)$ strongly disagree to often finding exactly what they search for on Google Scholar. This shows that the majority of respondents do not find exactly what they search for on Google Scholar.

Table 5 also shows that $21(9.4 \%)$ respondents strongly agree that Google Scholar usually provides details and in-depth information to them. Of the respondents, $92(41.2 \%)$ also agree with the same statement. However, 88 (39.5\%) disagree and $22(9.9 \%)$ strongly disagree. This result shows that there is much difference between those agreeing and disagreeing with the above statement.

TABLE 4: Perceived usefulness of Google Scholar

\begin{tabular}{|c|c|c|c|c|c|c|c|c|}
\hline \multirow[t]{2}{*}{ Perceived usefulness } & \multicolumn{2}{|c|}{ SA } & \multicolumn{2}{|c|}{ A } & \multicolumn{2}{|c|}{ D } & \multicolumn{2}{|c|}{ SD } \\
\hline & $n$ & $\%$ & $n$ & $\%$ & $n$ & $\%$ & $n$ & $\%$ \\
\hline 1. Google Scholar enables quick completion of research & 36 & 16.1 & 74 & 33.2 & 92 & 41.3 & 21 & 9.4 \\
\hline 2. Google Scholar makes research work easier & 29 & 13.1 & 52 & 23.3 & 93 & 41.7 & 49 & 21.9 \\
\hline 3. Using Google Scholar enhances my searching effectiveness & 25 & 11.2 & 98 & 43.9 & 84 & 37.7 & 16 & 7.2 \\
\hline 4. I can find many relevant articles with one search in Google Scholar & 31 & 13.9 & 93 & 41.7 & 77 & 34.5 & 22 & 9.9 \\
\hline 5. The resources in Google Scholar relate well to my research & 31 & 13.9 & 96 & 43.1 & 54 & 24.2 & 52 & 23.3 \\
\hline
\end{tabular}

SA, strongly agree; A, agreed; D, disagree; SD, strongly disagreed.

TABLE 5: Comprehensiveness and subjective norm.

\begin{tabular}{|c|c|c|c|c|c|c|c|c|}
\hline \multirow[t]{2}{*}{ Comprehensiveness and subjective norm } & \multicolumn{2}{|c|}{ SA } & \multicolumn{2}{|c|}{ A } & \multicolumn{2}{|c|}{ D } & \multicolumn{2}{|c|}{ SD } \\
\hline & $n$ & $\%$ & $n$ & $\%$ & $n$ & $\%$ & $n$ & $\%$ \\
\hline 1. The resources on Google Scholar are easy to understand & 26 & 11.6 & 103 & 46.2 & 78 & 34.9 & 16 & 7.2 \\
\hline 2. Google Scholar has enough resources for my study & 106 & 47.5 & 28 & 12.6 & 74 & 33.2 & 15 & 6.7 \\
\hline 3. Google Scholar covers a wide range of topics in my particular interest & 45 & 20.2 & 91 & 40.8 & 74 & 33.2 & 13 & 5.8 \\
\hline 4. I often find exactly what I search for while using Google Scholar & 16 & 7.2 & 34 & 15.2 & 126 & 56.5 & 47 & 21.1 \\
\hline 5. Google Scholar usually provides detail and in-depth information & 21 & 9.4 & 92 & 41.2 & 88 & 39.5 & 22 & 9.9 \\
\hline
\end{tabular}

SA, strongly agree; A, agreed; D, disagreed; SD, strongly disagreed. 


\section{Research Question 5}

What is the satisfaction level of postgraduate students of the University of Ilorin with Google Scholar platform?

The results in Table 6 show satisfaction levels of the postgraduate students of the University of Ilorin with the use of Google Scholar. Of the 223 respondents, 58 (26.1\%) strongly agree, $86(38.6 \%)$ agree, $53(23.7 \%)$ disagree and $26(11.6 \%)$ strongly disagree that they made the correct decision to use Google Scholar. This shows that the majority of respondents agree that they made the correct decision to use Google Scholar. Also, 85 (38.1\%) respondents strongly agree that they are satisfied with the results received from Google Scholar, and 120 (53.8\%) also agree with this statement, but $15(6.7 \%)$ disagree and $3(1.4 \%)$ strongly disagree of being satisfied with the results received from Google Scholar. With this, it is clear that the majority of respondents are satisfied with the results received from Google Scholar. The table also shows $112(16.6 \%)$ respondents strongly agree and indicate overall satisfaction with Google Scholar and 95 (42.6\%) of them also agree with the same statement. However, 91 (50.2\%) respondents disagree while 12 (5.4\%) strongly disagree to being satisfied with Google Scholar. This shows that a considerable majority of the respondents are satisfied with Google Scholar.

\section{Research Question 6}

What is the loyalty and intention of postgraduate students of the University of Ilorin towards the use of Google Scholar platform?

\section{Discussion of findings}

The results demonstrate that the majority of respondents strongly agree and agree they are aware of Google Scholar and usually make use of it. This finding is in accordance with the findings of Hamid and Asadi (2010) that scientists are becoming more aware of the quantity of scholarly papers searchable by Google; they are increasingly relying on Google for finding scholarly literature. The fact that Google Scholar usually meets the information needs of the research might be the reason for its popularity and its awareness on the part of the respondents in this study. Any platform or site where users get what they want and provide the information the users want do not need any advertisement because that is enough to advertise itself. This is the case of Google Scholar as revealed in this study.

The results in this study show that majorities have trust in Google Scholar as a platform reliable for searching scholarly articles. This corroborates the earlier report by Shen (2012) whose results demonstrate that, on average, respondents found Google Scholar easy to use and access, and that Google Scholar is perceived as a useful resource for their research because it enhanced the respondents' searching effectiveness. Undoubtedly, Google Scholar is a veritable search tool; therefore, it is unexpected that the respondents in this study regarded it as being reliable for scholarly searching and it improves their search effectiveness.

The results in this study revealed continuous intention of using Google Scholar. This is also in support of the findings by Cothran (2011) who indicated that several factors influence graduate students' intention to use Google Scholar, including students' perceived usefulness of Google Scholar, their sense of loyalty towards the search engine and its perceived ease of use. As observed from the literature, any information system that is perceived as useful will attract continuous use by users. So, any information that is easier to use and access will attract increased patronage by the users. Therefore, the indication of continuous intention to use Google Scholar by the respondents in this study is not surprising. The argument by Felter (2005) that most researchers preferred the simplicity of Google and would likely opt for Google Scholar over many more capable, but complicated, databases may also be the rationale for the intention of the respondents in this study to use Google Scholar.

The findings in this study also reported the overall satisfaction with Google Scholar by the respondents. Undoubtedly, its usefulness in terms of providing relevance information, meeting users' needs, ease of use and access, and its perception of being useful by the respondents may be the reason for the overall satisfaction the respondents in this study indicated with Google Scholar.

The results also indicate respondents do not agree that Google Scholar makes research quicker and easier. There is no good thing without the side effect or shortcoming. Google Scholar is no exception. No wonder, Henderson (2005) tested the search capabilities of Google Scholar and found a ranking bias towards older articles that had, as a result of the passage of time, been cited the greatest number of times. Henderson (2006) also lamented that Google Scholar lacked the standard Google search features - 'Similar pages' and 'Did you mean' features for alternative spellings. On the other hand, Golderman and Connolly (2007) applauded the compatibility of Google Scholar with bibliographic software applications such as Endnote and RefWorks, but faulted Google Scholar for failing to include search histories, alert services and utilities for sorting, marking and saving results.

TABLE 6: Satisfaction of postgraduate students with Google Scholar.

\begin{tabular}{|c|c|c|c|c|c|c|c|c|}
\hline \multirow[t]{2}{*}{ Satisfaction } & \multicolumn{2}{|c|}{ SA } & \multicolumn{2}{|c|}{ A } & \multicolumn{2}{|c|}{ D } & \multicolumn{2}{|c|}{ SD } \\
\hline & $n$ & $\%$ & $n$ & $\%$ & $n$ & $\%$ & $n$ & $\%$ \\
\hline 1. I think I made the correct decision to use Google Scholar & 58 & 26.1 & 86 & 38.6 & 53 & 23.7 & 26 & 11.6 \\
\hline 2. In general, I am satisfied with the results I received from Google Scholar & 85 & 38.1 & 120 & 53.8 & 15 & 6.7 & 3 & 1.4 \\
\hline 3. Overall, I am satisfied using Google Scholar & 112 & 50.2 & 95 & 42.6 & 12 & 5.4 & 4 & 1.8 \\
\hline
\end{tabular}

SA, strongly agree; A, agreed; D, disagreed; SD, strongly disagreed. 


\section{Recommendations}

Based on the findings of this study, the following recommendations were made: There is a need for orientation programmes to be held to increase the level of awareness of people about Google Scholar and also make them understand the advantages of using Google Scholar via a library medium. One of the advantages of using Google Scholar via the library medium is that it makes available to users restricted access articles that have been subscribed for. Training should also be conducted to enhance searching on Google Scholar so as to assist researchers in making their research work easier and quicker.

There is also a need to include an 'Ask-a-Librarian' feature on Google Scholar so that it will assist users in retrieving exactly what they search for. This will in turn increase the satisfaction level of the postgraduate students using Google Scholar and increase the trust they have in using Google Scholar.

\section{Conclusion}

The study has examined the usefulness of Google Scholar for the postgraduate students of the University of Ilorin. So far, the results generally indicated that the postgraduate students are not only aware of Google Scholar but also that they can access it to get scholarly articles and literature. However, the respondents are not satisfied with using Google Scholar as it does not speed up their research nor does it make their research easier. Google Scholar was considered useful because it covers broad topics in the area of interest, and they also find relevant articles related to their search using Google Scholar. The flaws noted in this study are also relevant to those noted by Jasco (2005) when he stated that Google Scholar lacks sophisticated searches that can help users retrieve exactly what they search for.

\section{Acknowledgements}

We acknowledged all authors whose works were cited in this article. We also appreciate the Department of Information Science, UNISA for the funding of the publication of this article.

\section{Competing interests}

The authors declare that they have no financial or personal relationships that may have inappropriately influenced them in writing this article.

\section{Authors' contributions}

T.A. was involved in the writing and typing of the report for this article. O.M. collected the data that was used in this study while T.A. assisted in the analysis of the data collected in this study.

\section{References}

Adlington, J. \& Benda, C., 2006, 'Checking under the hood: Evaluating Google Scholar for reference use', Internet Reference Services Quarterly 10(3-4), 135-148.

Bakkalbasi, N. \& Bauer, K., 2005, 'An examination of citation counts in a new scholarly communication environment', D-Lib Magazine 11(September), 1-10, viewed 23 July 2016, from http://www.dlib.org/dlib/september05/bauer/09bauer.html

Bakkalbasi, N., Bauer, K., Glover, J. \& Wang, L., 2006, 'Three options for citation tracking: Google Scholar, Scopus and Web of Science', Biomedical Digital Libraries 3, viewed 22 August 2015, from http://www.bio-diglib.com/content/3/1/7

Belkin, N.J. \& Croft, A., 1992, 'Anomalous state of knowledge as the basis for information retrieval', Canadian Journal of Library and Information Science 5 , 133-143.

Callicott, B. \& Vaughn, D., 2005, 'Google Scholar vs. library scholar: Testing the performance of Schoogle', Internet Reference Services Quarterly 10(3-4), 71-88. https://doi.org/10.1300/J136v10n03_08

Cothran, T., 2011, 'Google Scholar acceptance and use among graduate students: A quantitative study', Library \& Information Science Research 33, 293-301.

Felter, L.M., 2005, 'Google Scholar, Scirus, and the scholarly search revolution', Searcher 13, 43-48, viewed 23 July 2015, from http://www.scirus.com/press/pdf/ searcher_reprint.pdf

Golderman, G. \& Connolly, B., 2007, 'Who cited this?', Library Journal 132, 18-26.

Hamid, R. \& Asadi, J.S., 2010, 'Google and the scholar: The role of Google in scientists' information seeking behavior', Online Information Review 34(2), 282-294. https://doi.org/10.1108/14684521011036990

Henderson, J., 2005, 'Google Scholar: A source for clinicians?', CMAJ 172, 1549-1550. https://doi.org/10.1503/cmaj.050404

Henderson, J., 2006, Google Scholar goes to school the presence of Google Scholar on college and University web sites, viewed 12 March 2016, from https://www. academia.edu/4056717/Google_Scholar_Goes_to_School_The_Presence_of_ Google_Scholar_on_College_and_University_Web_Sites

Jasco, P., 2005, The pros and cons of computing the h-index using Google Scholar, University of Hawaii, Honolulu, $\mathrm{HI}$.

Kousha, K. \& Thelwall, M., 2006, 'Google Scholar citations and Google Web/URL citations: A multi-discipline exploratory analysis', Proceedings International Workshop on Webometrics, Informetrics and Scientometrics \& Seventh COLLNET Meeting, Nancy, France, viewed 22 July 2015, from http://eprints.rclis.org/ Meeting, Nancy, Fr
archive/00006416/

Mikki, S. 2009, 'Google Scholar Compared to Web of Science: A Literature Review', Nordic Journal of Information Literacy in Higher Education 1(1), 41-51.

Meho, L. \& Yang, K., 2007, 'A new era in citation and bibliometric analyses: Web of Science, Scopus, and Google Scholar', Journal of the American Society for Information Science and Technology 58:1-21, viewed 30 April 2015, from http:// arxiv.org/ftp/cs/papers/0612/0612132.pdf

Neuhaus, C., Neuhaus, E., \& Asher, A., 2008, 'Google Scholar goes to school: The presence of Google Scholar on college and university web sites', Journal of Academic of Librarianship 34(1), 39-51.

Neuhaus, C., Neuhaus, E., Asher, A. \& Wrede, C., 2006, 'The depth and breadth of Google Scholar: An empirical study', Portal: Libraries and the Academy 6(2), 127-141. https://doi.org/10.1353/pla.2006.0026

Noruzi, A., 2005, 'Web impact factor for Iranian universities', Webology 2(1), Article 11, viewed 12 March 2016, from: http://www.webology.ir/2005/v2n1/a11.html

Noruzi, A., 2006, 'Google Scholar: The new generation of citation indexes', Libri 55, 170-180, viewed 23 July 2015, from http://eprints.rclis.org/archive/00005542/01/ Google_Scholar,_The_New_Generation_of_Citation_Indexes.pdf

Shen, L., 2012, 'Graduate students report strong acceptance and loyal usage of Google Scholar', Evidence Based Library and Information Science 7(4), 96-98. https://doi. org/10.18438/B8RW3P 